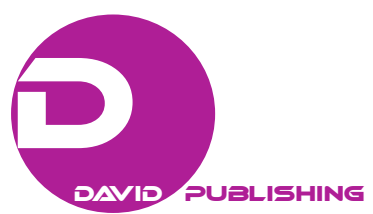

\title{
Follow the Open Source Road: Contemplating the Ethics of the New Information Ecology*
}

\author{
Andrew Jon Longcore \\ Indiana University of Pennsylvania, Indiana, PA, USA
}

\begin{abstract}
The evolutionary complexity of modern media systems represents an area of research that would benefit from further description and debate. In order to better understand the challenges and considerations associated with the new, constantly changing media ecology, a more holistic worldview must be catapulted to the forefront of the collective discussion. To that effect, the following essay examines the open source software movement from the philosophical perspective of Tibetan Buddhism. Using the principles of interconnectedness, impermanence, and the illusory nature of self and reality, all fundamental elements of Buddhist philosophy, this discussion provides a renewed framework for exploring the ethical implications of open sourcing. A review of Buddhist teachings, coupled with literature from foundational media theorists such as Neil Postman and Marshall and Eric McLuhan, will set the stage for contemplating the ethics of the modern information ecology, epitomized by the collaborative spirit and community connections of open source software.
\end{abstract}

Keywords: media ecology, Buddhism, open source software, information ethics

\section{Introduction}

"I touch my palms to water, leaving your fingerprints"

- From the poem Silent Resonance by Dale Winslow

(The Medium is the Muse: Channeling Marshall McLuhan)

Scholars throughout history have prophesized about the transformational changes that emerge from developments in media technology. From Gutenberg to Google, technological evolution has often created (or coincided with) cultural revolutions, rippling through the socio-political landscape to areas of education, economics, and beyond. The rapid pace at which modern development occurs has led to a surge in scholarly research on digital communication technology and social media platforms (Kuhn, 2007; Levinson, 2012; Scolari, 2012). However, another force is beginning to emerge in the debate over communication media, one that has remained in relative obscurity throughout much of the academic literature. That force is the spirit of open sourcing, an innovative approach to organizational networking and strategic planning that is poised to revolutionize institutions both public and private. These values, it should be noted, are prevalent throughout the philosophical writings of Buddhism, and represent an ethical framework for understanding the ongoing evolution of our complex global economy.

\footnotetext{
* The article was presented at the 16th Annual Convention of the Media Ecology Association (June 2015) at the Metropolitan State University, Denver, Colorado.

Andrew Jon Longcore, Instructor/Ph.D. Candidate, Department of Communications Media, Indiana University of Pennsylvania.
} 
The following discussion incorporates the ethical principles of Eastern spiritual philosophies (specifically Buddhism) into an exploration of the structure and behavior of modern interconnected media systems, specifically the open source software movement. Echoing the ideas and implications of systems thinking and chaos theory, Buddhism offers tremendous (and relatively untapped) insight into the constant, systemic struggle for self-advancement and a sense of control in a complex, interconnected network of change and impermanence. A brief summary of the open source software movement will set the stage for introducing the philosophical tenets of Tibetan Buddhism. After comparing the ethical principles of Buddhism to those associated with teleological ethics and utilitarian theory, the final section will contemplate the influential forces that continue to re-shape and re-create a dynamic web of interconnected media. A journey into the "Wild Wild West" of electronic media will lay the groundwork for future research into the value of Buddhist ethics in the development of open source software systems.

\section{Background and Context}

In many ways, this discussion explores the ethics of epistemology, or the study of knowledge. What is knowledge? Who (or what) should be allowed to access the information that leads to knowledge? If the public has a right to know, then at what point does additional information become irrelevant? To conceptualize and contemplate these complex probes, the following discussion considers the developments in communications media that have catalyzed a new configuration of the modern information ecology. Technologies that are included in this investigation represent important evolutionary advancements in the concept of "media". The first, collaboration-based "wikis", comprises the structural communities of the new cyberspace revolution. The second, "open source software" epitomizes the underlying ethics of wiki-based software and other new media technologies.

The shared spirit of open source software is representative of a broader cultural ethos, one with dramatic implications for the future of commercial media and corporate-controlled communication channels. Those implications are starting to have significant influence in our socio-political realities, as Sayogo et al. (2013) discuss in the following quote:

Governments around the world are promoting innovations to influence behavior of consumers, businesses, and other interest groups by implementing information policies that take advantage of new technologies and the vast stores of data being created in both public and private sector organizations. (Sayogo et al., 2013, p. 2)

The collaborative spirit that drives the decisions of governments, companies, and other institutions to open their intellectual property to collective settlement, so to speak, is the embodied ethic at the heart of the new information ecology, where open source communities and collaborative wikis are paving the way towards a new, truly participatory knowledge economy.

\section{The Open Source Revolution and the "Hacker Ethic"}

As Mader (2008) notes, "The web is becoming a place for the collaborative construction of information on an incredible scale, and the wiki is at the center of this transformation" (p. 5). Perhaps the most recognizable configuration of the wiki platform is the online archive Wikipedia. Indeed, Wikipedia is often misrepresented as synecdoche for the broader concept of wikis, but the former is only one example of the wiki, which is representative of a broader organizational style built on collaboration and co-creation of information and innovation. "The basic idea behind a wiki is that anyone who can view a page can just as easily edit it and save 
his or her changes" (Mader, 2008, p. 4). The difference between wikis and other alternative content management systems comes from structural variations between platforms (Mader, 2008; Tapscott \& Williams, 2008). Contrary to the hierarchical structure of traditional online media outlets and content management systems, wikis are non-hierarchical, representing a flatter and more democratic foundation. In many ways, the wiki is a step towards a continual transformation in the traditional concept of the information ecology.

Milberry and Anderson (2009) provide further insight into the open source revolution (of which the wiki platform is an integral and iconic figure), tracing the lineage to the early days in the history of the Internet. The idea of open sourcing is often attributed to the intrepid and idealistic researchers at the Massachusetts Institute of Technology and the birth of the Free Software Movement. These early pioneers in the exploration of cyberspace advocated a "hacker ethic", which the authors describes as a set of values based on the premise that "information is not a commodity to be bought and sold like any other good in a market economy, but a public good, collaboratively produced and freely circulated based on need and interest" (Milberry \& Anderson, 2009, p. 404). A similar spirit is echoed in the narrative presented by Sayogo et al. (2013), as the authors conceptualize the ethics of open source software within the framework of ethical consumerism. "By having information readily available, consumers could verify the company's commitment to ethical and sustainable conduct, thus enabling them to make informed purchasing decisions" (p. 4). The ability to access such contextual information, the authors note, is an important foundation of the ethical principle professed by "rational choice theory" (Sayogo et al., 2013, p. 4). Without the panacea of potentially relevant information, consumers have virtually zero chance (and thus very little opportunity) to act in truly rational ways in terms of their consumption patterns. From the perspective of rational choice theory, the nonexistence of access to all necessary information, a common complaint that arises with more traditional corporate media conglomerates, is itself an unethical system.

Championing a similar message as McLuhan (1972), Sayogo et al. (2013) explain how, "Consumer purchasing behavior in turn influences company conduct, ideally by increasing emphasis on ethical and sustainable models of production and operation" (p. 4). Unfortunately, the "commons ethos" that defines open sourcing, while seemingly rational on the surface, not to mention smart strategy for organizational management and competitive advantage, stands at odds with a dynamic cyberspace that is becoming increasingly consolidated under corporate control. Although platforms such as Google and Facebook offer "free" participatory services, Milberry and Anderson (2009) expose an important and often overlooked caveat:

The insidious combination of capitalist ownership at the infrastructural layer and corporate control at the content layer portends an impoverished future for the Internet: instead of a virtual commons, cyberspace will increasingly resemble private property, hemmed in and protected by state and market forces. (Milberry \& Anderson, 2009, p. 395)

The concentration of information under consolidated corporate controls has led to an "enclosure" of the online commons. The following quote helps to underscore the challenges associated with enclosure of media ownership:

Major online property holders such as Google and Facebook now have the power, and increasingly the incentive, to streamline their media holdings toward the efficient delivery of online participants to marketers and advertisers while narrowing the user experience to a singular corporate slice of cyberspace. (Milberry \& Anderson, 2009, p. 397)

The above quote highlights some of the multiple factors that influence the evolutionary development of open source software, particularly the ownership of the code and the compatibility of the software (location 
within the "stack" or embedded within the cyber-chain of logistics). As Milberry and Anderson (2009) argue, open source software represents a clarion call to challenge the commercialization and control of information that has occurred within the evolving media ecology. The path appears to be leading away from strategies of knowledge management and towards more collaborative knowledge communities.

The self-organization that occurs in open communities (Mader, 2008; Milberry \& Anderson, 2009) offers the potential solution to the complex requirements of modern global markets. To understand the ethical challenges and accolades that are embedded within open source software, it is necessary to position open source software on the broader continuum of media. In other words, how did the idea of "open sourcing" evolve to its present state? What are the implications of "open sourcing", as a personal and professional principle?

In many ways, open source software is the natural progression of an evolving landscape of electric media. This dynamic media movement is an extension of the human right to access information, a value described in terms of mass collaboration by Tapscott and Williams (2008):

It's an ethic that defines what the new web is becoming: a massive playground of information bits that are shared and remixed openly in a fluid and participatory tapestry. Having matured beyond its years as a static presentation medium, the Web is now the foundation for new dynamic forms of community and creative expression. Throw in a healthy dose of grassroots entrepreneurship and you have a potent recipe for economic revolution-a revolution that affects not just the obvious targets such as media, entertainment, and software, but is increasingly sweeping across all industries and sectors as mass collaboration makes inroads into activities ranging from science to manufacturing. (Tapscott \& Williams, 2008, p. 36)

The "open source" system embodies the spirit of Socrates, extending the dialogic method of investigation by inquiry in order to generate new insights and information. In practical terms, the open source revolution has forced a paradigm shift in corporate and organizational management. Adapting the Socratic paradox of knowledge, individuals in positions of institutional power must learn to "know what they don't know". By opening up the secret sources of organizational opportunity, corporate communication professionals create more opportunities for user-generated content. Content, in this context, may come in the form of value-based product (such as a popular web video, well-written prose, or a wellspring of poetic beauty). Content may also come in the form of criticism, which carries its own set of values (arguably more so than the former).

What are the ethical implications, in terms of both philosophical perspective and practical application, of "opening" the sources of organizational knowledge and content? Tapscott and Williams (2008) conceptualize the "ethics" of open source by discussing the fundamental values of "reciprocal sharing" and "collaborative discovery" (p. 171). These values, it should be noted, are prevalent throughout the philosophical writings of Buddhism, and represent an ethical framework for understanding the ongoing evolution of our complex global economy. Before discussing the virtues and values associated with Buddhism (as a specific set of ethical principles) we must begin with a caveat regarding ethics (in the present context).

Theorists and theologians throughout time have interpreted the concept of ethics in a multitude of different ways. From the deontological to the utilitarian, and the consequentialist discourses in between, each perspective has a relative value for the study of information ethics and the evolution of the modern information ecology. However, it is important to emphasize the relative measurement of value associated with various ethical prescriptions. For the purposes of the present discussion, then, the author employs his personal preference for the philosophical principles of Buddhism. 


\section{Conceptualizing the Basics of Buddhism}

The basic truths put forth by Tibetan Buddhism, as well as the practical guidelines prescribed by Buddhist meditations, offer a perfect framework within which a contemplation of the ethics of online media (in general) and open source software (in particular) may take place. The Sakya Monastery of Tibetan Buddhism provides a thoughtful and comprehensive snapshot of the values of Buddhism:

Buddhism is a tolerant religion that places emphasis on practical methods for cultivating spiritual awareness and on the importance of finding the truth for oneself. It treasures loving-kindness, compassion, equanimity, clarity of mind, and wisdom. ("Intro to Tibetan Buddhism", 2014, Para. 5)

The importance of mindfulness in the discussion of Buddhist ethics cannot be overstated. To that effect, one of the fundamental "truths" of Buddhist philosophy is the notion that the "self" is just an illusion, a perceptual product of the mind. The cognitive configuration of illusions created by the mind (as medium) often replaces the underlying "truth" or "reality" of the situation. Ricard and Thuan (2009) note a similar concept, explaining how, "Buddhism understood that the world we perceive is a mental reconstruction of exterior reality" (p. 121). Applying Buddhist philosophy to human nature, Emmanuel (2013) explains how "a person is merely a collection of psycho-physical elements or "aggregates" (khandhas) - body, feelings, perceptions, volitions, consciousness - that give rise to a causal pattern we identify as a particular individual" (p. 6). Beyond the individual level of analysis, the symbiotic insights contained within the teachings of Tibetan Buddhism have been applied to various aspects of social and corporate life, as epitomized in the following quote from Purser and Loy (2013):

Many corporate advocates argue that transformational change starts with oneself: if one's mind can become more focused and peaceful, then social and organizational transformation will naturally follow. The problem with this formulation is that today the three unwholesome motivations that Buddhism highlights — greed, ill will, and delusion — are no longer confined to individual minds, but have become institutionalized into forces beyond personal control. (Purser \& Loy, 2013, p. 2)

Additionally, Buddhist principles can be, have been, and certainly should be applied to the realm of global economics (Prayukvong \& Foster, 2014; Sivasai, 2012). Sivasai (2012), for instance, points to an important ethical dilemma that arises from increasing interconnected complexity. "As the scale of the economy grows, it also becomes increasingly difficult for us to know the effects of our actions on nature or on other people" (Sivasai, 2012, p. 32). At the same time, however, "the structures and institutions on which we depend are reifications of ignorance and greed - a denial of interdependence and impermanence" (p. 32). It is this incongruous juxtaposition that offers an area worthy of (and lacking in) critical investigation, and paradoxically provides the context for achieving congruence within the information ecology that surrounds the evolution of open source software.

\section{Connecting Buddhism and Information Ethics}

Perhaps the most explicit connection between Buddhism and the media configuration of the new information ecology can be found in the concept of connectivity itself. That is, the interconnectedness that is inherent in the structures of mass communication environments parallels one of the fundamental elements of the Buddhist conception of self, reality, and the nature of existence. Emmanuel (2013) reflects on the cosmological perspective of Buddhism, noting that, "All phenomena arise within a complex network of 
mutually conditioning causes and effects" (p. 6). Buddhism, far more so than traditional Western discourse, "includes deep respect for the natural world, in the belief that all life is interrelated and interdependent" (Emmanuel, 2013, p. 1). With that in mind, it is important to remember that our knowledge of such a vast complex system of interconnected parts will always be limited to the information we are able to access (and the bits of knowledge to which we focus our attention.

Ricard and Thuan (2009) illuminate a similar consideration in their discussion of the intersection of Buddhism and quantum physics. Referencing Godel's incompleteness theorem, the authors explain how "we are always limited in our knowledge of any system, such as the system we call the universe, while we are ourselves a part of that system" (Ricard \& Thuan, 2009, p. 35). The danger with this "incomplete" knowledge, then, is found in those systems that extend the illusory desires to control and bring order to an unpredictably and chaotic world. That is, traditional media corporations represent the structural extensions and "reifications of ignorance and greed - a denial of interdependence and impermanence" (Sivasai, 2012, p. 32). The desire for control is a by-product of the traditional hierarchical forms of knowledge management. Such desire, however, leads to a systematic oversight of the true nature within which we operate.

In order to better understand "reality," as conceptualized from the Buddhist perspective, we must follow the path towards another fundamental "truth" of Tibetan Buddhism. As Harvey (2010) argues, "Buddhism emphasizes that change and impermanence are fundamental features of everything, barnirvāna" (p. 34). Impermanence, in this context, relates to a striking incongruity between the Buddhist principle of life (and, by extension, the existence of material representations of reality) as impermanent and chaotic, and the evolution of the physical infrastructure of traditional media (such as newspapers, book publishers, radio stations, and television networks). Ricard and Thuan (2009) relate the concept of impermanence to the idea of organizational change and innovation, an interpretation that offers important insight for both the practice and pedagogy of Buddhist ethics:

At each infinitesimal moment, everything that seems to exist changes. Once we have recognized that change is inevitable and omnipresent, we can understand that the universe is not made up of solid, distinct entities, but of a dynamic flow of incessant interactions. (Ricard \& Thuan, 2009, pp. 101-102)

As the above quote makes clear, there is a connection between Buddhist philosophy and the study of "media ecology". To better understand the mechanism of mutual causality inherent in Buddhist scholarship, the following section provides an overview of the theoretical foundations of media ecology.

\section{Conceptualizing the New Information Ecology}

Scolari (2012) provides important context to qualify the term "ecology" within the present discussion of information ethics and open source software. Media ecology "tries to find out what roles media force us to play, how media structure what we are seeing or thinking, and why media make us feel and act as we do" (p. 205). Much like the contemplative aspects of mindfulness and meditation present in Buddhist philosophy, the study of media ecology involves an acknowledgement of the power of perception, at both the interpersonal and organizational levels. Summarizing the school of thought behind the field of media ecology, Scolari (2012) explains how the environments in which communication and interpersonal interaction take place "assign roles and pressure us to play them. Media environments specify what we can do and what we cannot" (p. 207). To that effect, M. McLuhan and E. McLuhan (1988) offer a path towards enlightening our understanding of how 
(and why) the ecology of open source software presents a fundamental threat to more traditional forms of media. Media "have no hierarchy or orderly sequence; they subsume, obsolesce, retrieve, extend each other, burrow on each other, hybridize, and miscegenate endlessly" (M. McLuhan \& E. McLuhan, 1988, p. 116). However, that is not to say these media operate without the influence of hierarchical pressures. Rather, many of the fundamental problems that exist in the current media landscape are the result of hierarchical imposition from outside forces.

It is this attempt from media moguls and power players to exert control over, manipulate, or otherwise command the circular complexity and user content of media systems that ultimately threatens their very existence as tools for interactive engagement and collaborative discourse. As Berkes et al. (2000) explain, the "Both knowledge and institutions require mechanisms for cultural internalization, so that learning can be encoded and remembered by the social group. World view or cosmology gives shape to cultural values, ethics, and the basic norms and rules of a society" (p. 1256). Indeed, the brick-and-mortar structures of digital technologies create numerous constraints on the availability, access, and application of various social media platforms. The physical systems upon which these technologies depend in order to operate are symbolic of the seemingly permanent obstacles that block the path towards true enlightenment.

Given that media are extensions of various aspects of the human condition, it seems appropriate that, as Milberry and Anderson (2009) allude, we should re-examine the commercial, corporate, and cultural forces that engender these rare but nonetheless constant cases of digital depravity. On the positive side, Gordon and Kittross (2013) explain, the rapidly-evolving forms of modern communications media have tremendous potential to foster and facilitate more collaborative and community-owned forms of content creation. "Emerging media and new information technologies may provide a better opportunity than do existing mass media operations to establish new practices and-more important-public policies that foster rather than discourage information equity" (Gordon \& Kittross, 2013, p. 114). Further demonstrating the potential for new media configurations to promote more democratic and participatory communities, and highlighting the importance of open source software as an opportunity for achieving a more ethical ecology of knowledge, Gordon and Kittross (2013) explain:

Since information is power in our increasingly information-based society some equality of access to it is crucial unless we are willing to settle for a society where some people or groups are disadvantaged because they can't obtain access. The risk is that society could become stratified on the basis of information available for people to use, as well as along economic, educational, racial, or other lines. (Gordon \& Kittross, 2013, p. 109)

To better understand the open source revolution, it is helpful to underscore the linguistic lineage of the most recognizable iteration of this social evolution - the open source software movement. As Vardouli and Buechley (2014) note, in the context of software, source code "is a set of instructions that can be executed into a software application" (p. 52). It seems logical that the "source code" referenced by the authors constitutes a metaphor that can be applied to other areas of our interconnected network of social and natural systems.

The open source movement extends well beyond the manufactured glass screen of the world of computer software, and represents an ethical spirit that flows through nearly every facet of our lives. What's more, the guiding spirit of the open source movement, as an ethical framework, is itself inherently interdisciplinary. Vardouli and Buechley (2014) explain the initial ethos behind the open software movement argued that 
"freedom to access, share and modify software's source code should be a fundamental human right" (p. 51). Interestingly, the authors discuss how the alternative path to supporting open source software adopted a utilitarian approach, arguing the beneficial impact of opening greater amounts of information to greater amounts of the "public". As the narrative put forth by Vardouli and Buechley (2014) suggests, the concept of open sourcing is rooted in the philosophical virtue of the public sphere.

The public sphere, in terms of the ethics of the new information ecology, can be conceptualized as the arena in which individuals (or users, in the parlance of cyberspace) have a guaranteed access to information that, in theory, will help them make better, more rational choices. Stein and Baines (2012), for example, explain how a public sphere advocates "public participation through discursive processes of deliberation" (p. 5). Indeed, the authors build upon the foundational concept of the public sphere put forth by Jurgen Habermas (1991) to construct a resonating argument related to discourse ethics. Further, the ethical discourse epitomized by a perfect public sphere (which the authors acknowledge is not always perfect in practice) parallels the implications produced by the open source software movement, implications that will dramatically influence the evolution of the new information ecology.

\section{Contemplating the Ethics of Open Source Software}

Buddhism seems to fall squarely under the categorical umbrella of teleological altruism, or the consequence-based school of ethics. Positioned in opposition to the egoism promoted by free-market ideologues, Buddhism argues for an altruistic contemplation that focuses on the consequences of one's actions. This altruistic notion is perhaps most easily conceptualized in the popular ethical theory of utilitarianism (Gordon \& Kittross, 2013), which places the focal point of ethical contemplation squarely in the public sphere. Utilitarian thinkers (such as philosopher John Stuart Mill) believe that ethical actions are those that produce the greatest "good" for the greatest number of people (while simultaneously limiting, or attempting to limit, the resulting amount of harm). Thus, it is the impact on others that can affect one's ethical decision-making patterns. In this regard, utilitarianism offers an ethical construct that echoes the teachings of Buddhism.

To achieve enlightenment means to inspire others to follow the same path. To decrease the collective suffering in the world, then, means to spark the discourse of discovery, to deliberate the collective decisions of our geo-political society in a dynamic environment. To that effect, it appears as though Buddhist monks and utilitarian theorists alike would agree that it is indeed ethical for online communities to invite their users to participate not just in the sustained use and operation of the platforms, but in their structural configurations and ethical evolutions. Further, given its deep and unyielding reverence for the natural ecology and an equally intense concern for actions that invariably influence the surrounding interconnected environment, Buddhism reflects the implicit ethical considerations associated with chaos theory.

In the midst of chaos, beauty can occur. Within the confines of McLuhan's metaphorical maelstrom, a solution lurks, hiding and meditating like a solitary denizen of the deep. It is precisely this element of chaos, commonly found in open systems, that enables the discovery of new insights and novel ways of thinking (Brafman \& Pollack, 2013). Although it may seem counterintuitive to invite all manner of call and response within a given open system or online community, it is often the devil in the details that leads to the discovery effective (and potentially more ethical) means for building a more dynamic and resilient system. 
An important and related ethical question that applies directly to the present discussion can be found in Levinson's (2012) detailed description of YouTube and other "new new media"- "Did the culture it [YouTube] created, in which anyone can be a star given the right (i.e. massively attractive) viral video, provide too easy an invitation to mentally unbalanced or ethically vacant people?" (p. 53). Further, as Levinson notes, "This is a question that arises whenever people as a whole are empowered by a new technology. The new device rarely creates the appetite for the evil deed" (Levinson, 2012, p. 53). Other theorists have argued a similar collective understanding of the ethical "truth" of media. Postman (1988), for example, summarizes the "symbolic relativist" stance on communication ethics:

Forms of communication are neither good nor bad in themselves. They become good or bad depending on their relationship to other symbols and on the functions they are made to serve within a social order. (Postman, 1988, p. 173)

Connecting the symbolic relativist position to the philosophical perspective of Buddhism, Ricard and Thuan (2009) explain how ethics, as a dichotomous relationship between judgments deemed "good" versus those deemed "bad", is itself a reflection of the interconnectedness of reality. In other words,

Good and evil exist only in terms of the happiness or suffering they create in ourselves or in other people. If we adopt a truly altruistic attitude, so that we are deeply concerned with the well-being of others, then this becomes the surest guide for our judgment. (Ricard \& Thuan, 2009, p. 19)

This, of course, begs the question: If determinations of ethics are based on the relationships of our actions (and our perceptions) to those of other individuals within our interconnected reality, how do we know what the term "well-being" actually means for other people?

\section{Additional Considerations}

Above and beyond the challenges associated with conceptualizing ethics, the element of complexity runs deep into the heart of ethics in the general sense. As the level of complexity increases within an organization or community of individuals, so too does the level of ethical complexity. In other words, as more individuals are involved in a given event or situation, the number of incongruities between individual ethical interpretations will likely increase. Complexity is also a key element in the open source revolution, as explained by Tapscott and Williams (2008):

When it comes to open source, complexity is both a feature and a bug. The three rules of open source-nobody owns it, everybody uses it, and anybody can improve it - may be the source of endless innovation, but they're equally a source of endless frustration... (Tapscott \& Williams, 2008, p. 86)

In the context of the open source movement, the potential for both collaborative creation and corporate commodification of information presents an area of constant ethical concern.

Sayogo et al. (2013) shine additional light on the growing collective need to recognize the value of open source software, and the related need to reconstruct our current "mass" of media platforms in order to facilitate more open and participatory progress:

...the growing intensity of market competition, increasing social pressures generated by mission-driven organizations and the public, and the economic value of information create market dynamics that motivate data producers to increase their transparency via open data as a competitive advantage. (Sayogo et al., 2013, p. 12)

The notion of transparency, epitomized by the "hacker ethic" and the open source software movement, has 
given rise to a growing collaborative commons that promotes a sort of "right to know", advocating more public freedom of accessing, sharing, and building upon information and innovations. With a stronger ethical foundation, such as the framework provided by Tibetan Buddhism (and supported with similar concepts from other Eastern religions and philosophical pathways), not even the sky is the limit with the possibility for discovering the next media.

If we can truly come to terms with the Buddhist belief in reality as impermanent and ever-changing and the self as perceptual costume, we can better guide our exploration into the evolving cyberscape of wiki-based platforms and open source software. Through the use of meditation and contemplative practice, we can incorporate the basic truths of Buddhism and the spirit of mindfulnessas a means of de-constructing the illusions of reality ingrained in traditional Western discourse. By developing more ethical strategies for capitalizing on the new information ecology, we may eventually find our way to ethical enlightenment.

\section{References}

"Intro to Tibetan Buddhism". (2014). The Sakya Monastery. Retrieved December 12, 2014, from http://www.sakya.org/introtibetanbuddhism.html

Berkes, F., Colding, J., \& Folke, C. (2000). Rediscovery of traditional ecological knowledge as adaptive management. Ecological Applications, 10(5), 1251-1262.

Brafman, O., \& Pollack, J. (2013). The chaos imperative: How chance and disruption increase innovation, effectiveness, and success. Crown Business.

Emmanuel, S. M. (2013). A companion to buddhist philosophy. In J. Wiley, A. D. Gordon, \& J. M. Kittross (Eds.), Controversies in media ethics (2nd ed.). New York, NY: Addison Wesley Longman, Inc.

Habermas, J. (1991). The structural transformation of the public sphere: An inquiry into a category of bourgeois society. Cambridge, Mass: MIT press.

Harvey, P. (2012). An introduction to Buddhism: Teachings, history and practices. Cambridge: Cambridge University Press.

Kuhn, M. (2007). Interactivity and prioritizing the human: A code of blogging ethics. Journal of Mass Media Ethics, 22(1), 18-36. Levinson, P. (2012). New new media (2nd ed.). Boston: Pearson (Penguin Academics).

Mader, S. (2008). Wikipatterns. Indianapolis, IN: John Wiley \& Sons.

McLuhan, M. (1972). Take today: The executive as dropout. Ontario, CA: Longman Canada Limited.

McLuhan, M., \& McLuhan, E. (1988). Laws of media: The new science. Toronto, CA: University of Toronto Press.

Milberry, K., \& Anderson, S. (2009). Open sourcing our way to an online commons: Contesting corporate impermeability in the new media ecology. Journal of Communication Inquiry, 33(4), 393-412.

Postman, N. (1988). Conscientious objections: Stirring up trouble about language, technology and education. New York, NY: Random House LLC.

Prayukvong, W., \& Foster, M. J. (2014). Buddhist economics meets corporate social responsibility. International Journal of Economics and Business Research, 8(2), 175-192.

Purser, R., \& Loy, D. (2013). Beyond McMindfulness. Retrieved December 10, 2014, from http://www. achtsame-wirtschaft.de/tl_files/netzwerk_achtsame_wirtschaft/pdf/Beyond-McMindfulness-Purser-Loy.pdf

Ricard, M., \& Thuan, T. X. (2009). The quantum and the lotus: A journey to the frontiers where science and Buddhism meet. Random House LLC.

Rinzler, L. (2007). The Buddha walks into a bar: Meditations for a new generation. Shambhala Publications.

Sayogo, D. S., Zhang, J., Pardo, T. A., Tayi, G. K., Hrdinova, J., Andersen, D. F., \& Luna-Reyes, L. F. (2013). Going beyond open data: Challenges and motivations for smart disclosure in ethical consumption. Journal of Theoretical and Applied Electronic Commerce Research, 9(2), 1-16.

Scolari, C. A. (2012). Media ecology: Exploring the metaphor to expand the theory. Communication Theory, 22(2), $204-225$.

Sivasai, C. (2012). Buddhism and economics: An analysis and reflection. Quest International Multidisciplinary Research Journal, 1(2), 32-38.

Stein, J., \& Baines, D. (2012). Myth-making on the business pages: Local press and global crisis. The International Journal of Communication Ethics, 9(1), 52-59. 
Strate, L., \& Karasick, A. (2014). The medium is the muse: Channeling Marshall McLuhan. Neopoiesis Press, LLC.

Tapscott, D., \& Williams, A. D. (2008). Wikinomics: How mass collaboration changes everything. New York: Penguin.

Vardouli, T., \& Buechley, L. (2014). Open source architecture: An exploration of source code and access in architectural design. Leonardo, 47(1), 51-55.

Watts, J., \& Loy, D. (1998). The religion of consumption: A Buddhist perspective. Development-Journal of the Society for International Development-English Edition, 1, 61-66. 